\title{
Multiattribute Decision Making Based on Entropy under Interval-Valued Intuitionistic Fuzzy Environment
}

\author{
Yingjun Zhang, ${ }^{1}$ Peihua Li, ${ }^{2}$ Yizhi Wang, ${ }^{1}$ Peijun $\mathrm{Ma},{ }^{3}$ and Xiaohong Su${ }^{3}$ \\ ${ }^{1}$ School of Computer and Information Technology, Beijing Jiaotong University, Beijing 100044, China \\ ${ }^{2}$ School of Information and Communication Engineering, Dalian University of Technology, Dalian 116024, China \\ ${ }^{3}$ School of Computer Science and Technology, Harbin Institute of Technology, Harbin 150001, China \\ Correspondence should be addressed to Yingjun Zhang; hitzyj@163.com
}

Received 9 March 2013; Accepted 30 July 2013

Academic Editor: Yi-Kuei Lin

Copyright (c) 2013 Yingjun Zhang et al. This is an open access article distributed under the Creative Commons Attribution License, which permits unrestricted use, distribution, and reproduction in any medium, provided the original work is properly cited.

\begin{abstract}
Multiattribute decision making (MADM) is one of the central problems in artificial intelligence, specifically in management fields. In most cases, this problem arises from uncertainty both in the data derived from the decision maker and the actions performed in the environment. Fuzzy set and high-order fuzzy sets were proven to be effective approaches in solving decision-making problems with uncertainty. Therefore, in this paper, we investigate the MADM problem with completely unknown attribute weights in the framework of interval-valued intuitionistic fuzzy (IVIF) set (IVIFS). We first propose a new definition of IVIF entropy and some calculation methods for IVIF entropy. Furthermore, we propose an entropy-based decision-making method to solve IVIF MADM problems with completely unknown attribute weights. Particular emphasis is put on assessing the attribute weights based on IVIF entropy. Instead of the traditional methods, which use divergence among attributes or the probabilistic discrimination of attributes to obtain attribute weights, we utilize the IVIF entropy to assess the attribute weights based on the credibility of the decisionmaking matrix for solving the problem. Finally, a supplier selection example is given to demonstrate the feasibility and validity of the proposed MADM method.
\end{abstract}

\section{Introduction}

Atanassov and Gargov extended the intuitionistic fuzzy (IF) set (IFS) to the interval-valued intuitionistic fuzzy (IVIF) set (IVIFS) [1-3], characterized by membership and nonmembership functions whose values are intervals rather than real numbers. Since IFS and IVIFS were proposed, a great deal of literature abounds on both theoretical research of IFS and IVIFS as well as on application research in various fields, such as image processing [4-6], pattern recognition [7-9], clustering analysis [10-12], supplier selection, and decision-making analysis $[11,13,14]$.

In particular, IVIFS and IFS are effective in solving the fuzzy decision-making problems. In most fuzzy multi-attribute decision making (MADM) problems, the preference over alternatives provided by decision makers is usually not sufficient for the crisp membership and nonmembership degree values, because things are fuzzy, uncertain, and probably influenced by the subjectivity of the decision makers, or the knowledge and data about the problem domain are insufficient during the decision-making process [13-17]. Therefore, the preferences among alternatives with uncertainty may be denoted by IFS or IVIFS for decision-making problems [7, 11, 13-15]. Chen and Tan [18], Xu [11, 19], Ye [20], Lakshmana Gomathi Nayagam et al. [21], and Wang et al. [22] proposed some methods to rank alternatives expressed with IVIFSs/IFSs and discussed their application in the MADM field. Atanassov [23] introduced some aggregation operators on IFS and IVIFS. After the pioneering work of Atanassov, some aggregation operators were proposed and utilized to tackle the MADM problems with uncertainty [24-33]. Xu and Yager [34], Li et al. [35-38], and Dubey et al. [15] constructed IF and IVIF MADM models based on optimization theories, such as linear, nonlinear, and fractional programming. Meanwhile, decision-making methods based on some measures (such as distance, similarity degree, correlation coefficient, and entropy) were proposed in dealing with fuzzy IF and IVIF MADM problems [7, 19, 35, 39-41]. In [13, 17, 22, 34, 37], 
emphasis was given that not only the information of alternatives on attributes may be fuzzy but also the attribute weight information may be partially known or unknown in some situations. In fact, proper assessment of attribute weights plays an important and essential role in the decision-making process, because the variation of weight values may result in different final ranking order of alternatives [11, 14, 17].

Generally speaking, the attribute weights in MADM can be classified as subjective and objective attribute weights based on the information acquisition approach [14]. The subjective attribute weights are determined by preference information on the attributes given by the decision maker, who provides subjective intuition or judgments on specific attributes. The objective attribute weights are determined by the decision-making matrix. Analytic hierarchy process (AHP) method [42] and Delphi method [16] are two classical methods for generating subjective attribute weights. In terms of determining objective attribute weights, one of the most famous approaches is the Shannon entropy method, which expresses the relative intensities of attribute importance to signify the average intrinsic information transmitted to the decision maker. So far, a lot of literature pertaining to MADM analysis under IF/IVIF environment has been published using subjective weights [11,15-17]. In the course of determining objective attribute weights under IF environment, Chen and Li [14] utilized IF entropy to assess the objective attribute weights in dealing with the IF MADM problem with completely unknown attribute weights. Despite existing research effort, solving the fuzzy MADM problems with completely unknown attribute weights in the framework of IVIFS remains an open problem $[11,14,17,35,37,38]$.

In an attempt to address such problems, we propose a novel entropy-based decision-making method under IVIF environment. Our focus is on the assessment of attribute weights. Meanwhile, we propose a definition of entropy on IVIFS, as well as a method to calculate it. Instead of using traditional methods, which use divergence among attributes or the probabilistic discrimination of attributes to obtain attribute weights, IVIF entropy is utilized to assess the objective weights based on the credibility of the input data. Furthermore, we construct a MADM model based on the IVIF weighted averaging (IIFWA) operator and the ranking functions on IVIFS.

The rest of this paper is organized as follows: in Section 2, we briefly review the concepts of IFS, IVIFS and some of their operations. In Section 3, we propose a definition of IVIF entropy and some calculation methods on entropy. An entropy-based MADM method within the framework of IVIFS is proposed in Section 4. A numerical example is utilized to illustrate the applicability of the proposed method in Section 5. Finally, conclusion is given in Section 6.

\section{Preliminaries}

In this section, we briefly review some basic concepts, for example, IFS, IVIFS, and their relevant operations.

Notation. $X=\left\{x_{1}, x_{2}, \ldots, x_{N}\right\}$ is the universal set (it is clear that $N=\operatorname{Cardinal}(X))$. FS $s(X), \operatorname{IFS} s(X)$, and $\operatorname{IVIFS} s(X)$ denote all the fuzzy sets on $X$, all the IFSs on $X$, and all the IVIFSs on $X$, respectively.

Definition 1 (see [1]). Let $X$ be a set. An IFS $A$ in $X$ is defined as

$$
A=\left\{\left(x, \mu_{A}(x), v_{A}(x)\right) \mid x \in X\right\},
$$

where

$$
\mu_{A}: X \longrightarrow[0,1], \quad v_{A}: X \longrightarrow[0,1]
$$

are two maps satisfying

$$
0 \leq \mu_{A}(x)+\nu_{A}(x) \leq 1, \quad \forall x \in X .
$$

$\mu_{A}(x)$ and $v_{A}(x)$ denote the membership and nonmembership degrees of $x$ to $A$, respectively. For each IFS $A$ in $X$, we designate

$$
\pi_{A}(x)=1-\mu_{A}(x)-v_{A}(x),
$$

an intuitionistic index of $x$ in $A$.

The following expressions are defined in $[1,11]$ for all $A$ and $B$ belonging to IFS $s(X)$ :

(1) $A^{c}=\left\{\left(x, v_{A}(x), \mu_{A}(x)\right) \mid x \in X\right\}$,

(2) $A \leq B$ if and only if $\mu_{A}(x) \leq \mu_{B}(x)$ and $\nu_{A}(x) \geq \nu_{B}(x)$ for all $x \in X$,

(3) $A \preceq B$ if and only if $\mu_{A}(x) \leq \mu_{B}(x)$ and $\nu_{A}(x) \leq \nu_{B}(x)$ for all $x \in X$,

(4) $A=B$ if and only if $A \leq B$ and $B \leq A$.

Definition 2 (see $[2,23])$. Let $X$ be a set and Int $[0,1]$ the set of all closed subintervals of $[0,1]$. An IVIFS $A$ in $X$ is defined as

$$
A=\left\{\left(x, \mu_{A}(x), v_{A}(x)\right) \mid x \in X\right\},
$$

where

$$
\mu_{A}: X \longrightarrow \text { Int }[0,1], \quad v_{A}: X \longrightarrow \text { Int }[0,1],
$$

for which

$$
0 \leq \sup \left(\mu_{A}(x)\right)+\sup \left(\nu_{A}(x)\right) \leq 1, \quad \forall x \in X .
$$

The intervals $\mu_{A}(x)$ and $\nu_{A}(x)$ denote the membership and nonmembership degrees of $x$ to $A$, respectively. Let

$$
A=\left\{\left(x,\left[\mu_{A_{L}}(x), \mu_{A_{U}}(x)\right],\left[v_{A_{L}}(x), v_{A_{U}}(x)\right]\right) \mid x \in X\right\},
$$

where $\mu_{A_{L}}(x)=\inf \left(\mu_{A}(x)\right), v_{A_{L}}(x)=\inf \left(\nu_{A}(x)\right), \mu_{A_{U}}(x)=$ $\sup \left(\mu_{A}(x)\right)$, and $v_{A_{U}}(x)=\sup \left(\nu_{A}(x)\right)$. If $\mu_{A_{L}}(x)=\mu_{A_{U}}(x)$ and $v_{A_{L}}(x)=v_{A_{U}}(x)$, then IVIFS $A$ reduces to an IFS.

For each IVIFS $A$, we designate

$$
\pi_{A}(x)=1-\mu_{A}(x)-v_{A}(x),
$$

an intuitionistic interval of $x$ in $A$, where

$$
\pi_{A}(x)=\left[1-\mu_{A_{U}}(x)-v_{A_{U}}(x), 1-\mu_{A_{L}}(x)-v_{A_{L}}(x)\right] .
$$


The following expressions are defined for all $A$ and $B$ belonging to IVIFS $s(X)[11]$ :

(1) $A^{c}=\left\{\left(x,\left[v_{A_{L}}(x), v_{A_{U}}(x)\right],\left[\mu_{A_{L}}(x), \mu_{A_{U}}(x)\right]\right) \mid x \in\right.$ $X\}$,

(2) $A \leq B$ if and only if $\mu_{A_{L}}(x) \leq \mu_{B_{L}}(x), \mu_{A_{U}}(x) \leq$ $\mu_{B_{U}}(x), v_{A_{L}}(x) \geq v_{B_{L}}(x)$, and $v_{A_{U}}(x) \geq v_{B_{U}}(x)$ for all $x \in X$,

(3) $A \preceq B$ if and only if $\mu_{A_{L}}(x) \leq \mu_{B_{L}}(x), \mu_{A_{U}}(x) \leq$ $\mu_{B_{U}}(x), v_{A_{L}}(x) \leq v_{B_{L}}(x)$, and $v_{A_{U}}(x) \leq v_{B_{U}}(x)$ for all $x \in X$,

(4) $A=B$ if and only if $A \leq B$ and $B \leq A$.

Ranking the alternatives expressed with IVIFSs is necessary to deal with the MADM problem within the framework of IVIFS. We introduce the score function and accuracy function of IVIFS proposed by $\mathrm{Xu}$ [11].

Definition 3 (see [11]). Let $\alpha=([a, b],[c, d])$ be an IVIF number. The score function $s$ of $\alpha$ is defined as follows:

$$
s(\alpha)=\frac{1}{2}(a+b-c-d),
$$

where $s(\alpha) \in[0,1]$.

Definition 4 (see [11]). Let $\alpha=([a, b],[c, d])$ be an IVIF number. The accuracy function $h$ of $\alpha$ is defined as follows:

$$
h(\alpha)=\frac{1}{2}(a+b+c+d)
$$

where $h(\alpha) \in[0,1]$

Based on the score function and the accuracy function, $\mathrm{Xu}$ further introduced an algorithm of ranking alternatives expressed with IVIFSs.

Definition 5 (see [11]). Let $\alpha=([a, b],[c, d])$ and $\beta=$ $\left(\left[a_{1}, b_{1}\right],\left[c_{1}, d_{1}\right]\right)$ be two IVIF numbers; then the ranking order between $\alpha$ and $\beta$ is defined as follows:

(1) If $s(\alpha)>s(\beta)$, then $\alpha>\beta$.

(2) If $s(\alpha)<s(\beta)$, then $\alpha<\beta$.

(3) If $s(\alpha)=s(\beta)$, then

(a) if $h(\alpha)>h(\beta)$, then $\alpha>\beta$;

(b) if $h(\alpha)<h(\beta)$, then $\alpha<\beta$;

(c) if $h(\alpha)=h(\beta)$, then $\alpha \sim \beta$.

\section{Entropy on IVIFSs}

The definition of entropy on IFSs/IVIFSs has a great importance in the theoretical research of IFSs/IVIFSs. In 1996, Burillo and Bustince [43] introduced the IF entropy for the first time, and, in 2001, Szmidt and Kacprzyk [44] proposed a non-probabilistic-type entropy measure for IFSs. Later, Hung [45] and Zhang et al. [46] constructed the IF entropy based on the distance measures among IFSs. Vlachos and Sergiadis [9] introduced the De Luca-Termini nonprobabilistic entropy for
IFSs. Zeng and Su [32] introduced the IF entropy based on the similarity among IFSs. Ye [47] constructed two IF entropies based on the fuzzy entropy established by Zadeh [48]. Chen and $\mathrm{Li}$ [14] presented different entropies on IFSs. So far, a great deal of literature on entropy on IFSs is available, but, unfortunately, to our knowledge, only a few works on entropy on IVIFSs exist. Two published papers are found in [49] which extended De luca-Termini axioms and proposed an IVIF entropy, and in [41] which proposed an IVIF entropy in dealing with multiple-attribute decision-making problem.

3.1. Entropy on IFSs. Based on the definition of fuzzy entropy [48], Burillo and Bustince [43] defined the IF entropy as follows.

Definition 6 (see [43]). A real function $I: \operatorname{IFS} s(X) \rightarrow R^{+}$is called an entropy if $I$ has the following properties:

(1) $I(A)=0$ if and only if $A \in \mathrm{FS} s(X)$,

(2) $I(A)=\operatorname{Cardinal}(X)=N$ if and only if $\mu_{A}(x)=0$ and $\nu_{A}(x)=0$ for all $x \in X$,

(3) $I(A)=I\left(A^{c}\right)$ for all $A \in \operatorname{IVIFS}(X)$,

(4) If $A \preceq B$, then $I(A) \geq I(B)$.

To construct the entropy on IFSs, Burillo and Bustince introduced function $\Phi: D \rightarrow[0,1]$, where $D=\{(x, y) \in$ $[0,1] \times[0,1] \mid x+y \leq 1\}$. Meanwhile, function $\Phi$ satisfies the following conditions:
(a) $\Phi(x, y)=1$, if and only if $x+y=1$,
(b) $\Phi(x, y)=0$, if and only if $x=0$ and $y=0$,
(c) $\Phi(x, y)=\Phi(y, x)$,
(d) if $x \leq x^{\prime}$ and $y \leq y^{\prime}$, then $\Phi(x, y) \leq \Phi\left(x^{\prime}, y^{\prime}\right)$.

According to Definition 6 and function $\Phi$, Bustince and Burillo proposed a theorem to construct the entropy on IFSs.

Theorem 7 (see [43]). Let $X=\left\{x_{1}, x_{2}, \ldots, x_{N}\right\}, I$ : $\operatorname{IFS} s(X) \rightarrow R^{+}$, and $A \in \operatorname{IFS} s(X)$. If $I(A)=\sum_{i=1}^{N}(1-$ $\left.\Phi\left(\mu_{A}\left(x_{i}\right), v_{A}\left(x_{i}\right)\right)\right)$, where $\Phi$ satisfies conditions $(a)-(d)$; then $I$ is an entropy on $A$.

3.2. Entropy on IVIFSs. Based on Definition 6 and some properties on IFS $[50,51]$, we define the entropy on IVIFSs as follows.

Definition 8. A real function $I: \operatorname{IVIFS} s(X) \rightarrow R^{+}$is called an entropy, if $I$ has the following properties:

(1) $I(A)=0$, if and only if $A$ is a fuzzy set;

(2) $I(A)=(1 / N) \operatorname{Cardinal}(X)=1$, if and only if $\mu_{A}(x)=$ $[0,0]$ and $\nu_{A}(x)=[0,0]$ for all $x \in X$;

(3) $I(A)=I\left(A^{c}\right)$ for all $A \in \operatorname{IVIFS}(X)$;

(4) If $A \preceq B$, then $I(A) \geq I(B)$. 
For any $A \in \operatorname{IVIFS}(X)$, we introduce the following operator:

$$
\begin{aligned}
& F_{p}(A)= \\
& \quad\left\{\left(x, \mu_{A_{L}}(x)+p \Delta \mu_{A}(x), v_{A_{L}}(x)+p \Delta \nu_{A}(x)\right) \mid x \in X\right\},
\end{aligned}
$$

where $\Delta \mu_{A}(x)=\mu_{A_{U}}(x)-\mu_{A_{L}}(x), \Delta v_{A}(x)=v_{A_{U}}(x)-v_{A_{L}}(x)$, and $p \in(0,1)$. Obviously, $F_{p}(A)$ is an IFS on $X$.

The following theorem gives an expression that allows us to construct different entropies on IVIFSs.

Theorem 9. Let $X=\left\{x_{1}, x_{2}, \ldots, x_{N}\right\}, I: \operatorname{IVIFS} s(X) \rightarrow R^{+}$, and $A \in \operatorname{IVIFS} s(X)$. If

$$
I(A)=\frac{1}{N} \sum_{i=1}^{N}\left(1-\Phi\left(\bar{\mu}_{A}\left(x_{i}\right), \bar{\nu}_{A}\left(x_{i}\right)\right)\right)
$$

then $I$ is an entropy of $A$, where $\Phi$ satisfies conditions (a)-(d), $\bar{\mu}_{A}(x)=\mu_{A_{L}}(x)+p \Delta \mu_{A}(x), \bar{v}_{A}(x)=v_{A_{L}}(x)+p \Delta v_{A}(x)$, and $p \in(0,1)$.

Proof. (1) $\Leftarrow$ If $A$ is a fuzzy set, then $\mu_{A_{L}}(x)=\mu_{A_{U}}(x)$, $v_{A_{L}}(x)=v_{A_{U}}(x)$, and $\mu_{A_{U}}(x)+v_{A_{U}}(x)=1$ for all $x \in X$. Clearly, $I(A)=0$ according to the condition (a) of $\Phi$.

$\Rightarrow I(A)=0$ implies that $\Phi\left(\bar{\mu}_{A}\left(x_{i}\right), \bar{v}_{A}\left(x_{i}\right)\right)=1(i=$ $1,2, \ldots, N)$ for any $p \in(0,1)$. If $A$ is not a fuzzy set, then $\mu_{A_{L}}\left(x_{i^{*}}\right)+v_{A_{L}}\left(x_{i^{*}}\right)<1$ for some $x_{i^{*}} \in X\left(i^{*} \in\{1,2, \ldots, N\}\right)$. As $\mu_{A_{L}}\left(x_{i^{*}}\right)+v_{A_{L}}\left(x_{i^{*}}\right)<1$ and $\mu_{A_{U}}\left(x_{i^{*}}\right)+v_{A_{U}}\left(x_{i^{*}}\right) \leq 1$, $\bar{\mu}_{A}\left(x_{i^{*}}\right)+\bar{\nu}_{A}\left(x_{i^{*}}\right)<1$ for any $p \in(0,1)$. According to conditions (a) and (d) of $\Phi$, we have $\Phi\left(\bar{\mu}_{A}\left(x_{i^{*}}\right), \bar{\nu}_{A}\left(x_{i^{*}}\right)\right)<1$. Thus, $I(A)>0$, which contradicts $I(A)=0$. Thus, $A$ is a fuzzy set when $I(A)=0$.

(2) $\mu_{A}(x)=[0,0]$ and $\nu_{A}(x)=[0,0]$ for all $x \in X$ which implies that $\bar{\mu}_{A}\left(x_{i}\right)=0, \bar{\nu}_{A}\left(x_{i}\right)=0(i=1,2, \ldots, N)$ for any $p \in(0,1)$. Based on condition (b) of $\Phi$, we have $I(A)=1$.

$\Rightarrow I(A)=1$ implies that $\Phi\left(\bar{\mu}_{A}\left(x_{i}\right), \bar{v}_{A}\left(x_{i}\right)\right)=0(i=$ $1,2, \ldots, N)$ for any $p \in(0,1)$. As $0 \leq \bar{\mu}_{A}(x)+\bar{\nu}_{A}(x) \leq 1$, $\bar{\mu}_{A}(x)+\bar{v}_{A}(x)=0$ based on the condition (b) of $\Phi$. If $x_{i^{*}} \in X$ exists that satisfies $\mu_{A_{U}}\left(x_{i^{*}}\right)+\nu_{A_{U}}\left(x_{i^{*}}\right)>0$, it means that $\bar{\mu}_{A}\left(x_{i^{*}}\right)+\bar{\nu}_{A}\left(x_{i^{*}}\right)>1$ for any $p \in(0,1)$. According to conditions (b) and (d) of $\Phi$, we have $\Phi\left(\bar{\mu}_{A}\left(x_{i^{*}}\right), \bar{\nu}_{A}\left(x_{i^{*}}\right)\right)>0$. Obviously, $I(A)<1$, which contradicts $I(A)=1$. Thus, we have $\mu_{A}(x)=[0,0]$ and $\nu_{A}(x)=[0,0]$.

(3) As $A^{c}=\left\{\left(x, v_{A}(x), \mu_{A}(x)\right) \mid x \in X\right\}$, clearly, $I(A)=$

(4) If $A \preceq B$, then $\mu_{A_{L}}(x) \leq \mu_{B_{L}}(x), \mu_{A_{U}}(x) \leq \mu_{B_{U}}(x)$, $v_{A_{L}}(x) \leq \nu_{B_{L}}(x)$, and $v_{A_{U}}(x) \leq \nu_{B_{U}}(x)$; then $(1-p)\left(\mu_{B_{L}}(x)-\right.$ $\left.\mu_{A_{L}}(x)\right)+p\left(\mu_{B_{U}}(x)-\mu_{A_{U}}(x)\right)+(1-p)\left(v_{B_{L}}(x)-v_{A_{L}}(x)\right)+$ $p\left(\nu_{B_{U}}(x)-v_{A_{U}}(x)\right) \geq 0$ for any $p \in(0,1)$; then $\mu_{A_{L}}(x)+$ $p\left(\mu_{A_{U}}(x)-\mu_{A_{L}}(x)\right)+v_{A_{L}}(x)+p\left(v_{A_{U}}(x)-v_{A_{L}}(x)\right) \leq \mu_{B_{L}}(x)+$ $p\left(\mu_{B_{U}}(x)-\mu_{B_{L}}(x)\right)+v_{B_{L}}(x)+p\left(v_{B_{U}}(x)-v_{B_{L}}(x)\right)$, that is, $\bar{\mu}_{A}(x)+$ $\bar{v}_{A}(x) \leq \bar{\mu}_{B}(x)+\bar{\nu}_{B}(x)$. According to condition (d) of $\Phi$, we have $\Phi\left(\bar{\mu}_{A}\left(x_{i}\right), \bar{v}_{A}\left(x_{i}\right)\right) \leq \Phi\left(\bar{\mu}_{B}\left(x_{i}\right), \bar{\nu}_{B}\left(x_{i}\right)\right)(i=1,2, \ldots, N)$, that is, $1-\Phi\left(\bar{\mu}_{A}\left(x_{i}\right), \bar{v}_{A}\left(x_{i}\right)\right) \geq 1-\Phi\left(\bar{\mu}_{B}\left(x_{i}\right), \bar{v}_{B}\left(x_{i}\right)\right)$ implying $I(A) \geq I(B)$.

The proof is completed.

\begin{tabular}{|c|c|c|c|c|c|c|c|}
\hline & & $A_{1}$ & $A_{2}$ & $A_{3}$ & $A_{4}$ & $A_{5}$ & $A_{6}$ \\
\hline \multirow{9}{*}{$I_{\Phi_{1, \text { cost }}}$} & $p=0.1$ & 1 & 0 & 0 & 0.5800 & 0.5800 & 0.1800 \\
\hline & $p=0.2$ & 1 & 0 & 0 & 0.5600 & 0.5600 & 0.1600 \\
\hline & $p=0.3$ & 1 & 0 & 0 & 0.5400 & 0.5400 & 0.1400 \\
\hline & $p=0.4$ & 1 & 0 & 0 & 0.5200 & 0.5200 & 0.1200 \\
\hline & $p=0.5$ & 1 & 0 & 0 & 0.5000 & 0.5000 & 0.1000 \\
\hline & $p=0.6$ & 1 & 0 & 0 & 0.4800 & 0.4800 & 0.0800 \\
\hline & $p=0.7$ & 1 & 0 & 0 & 0.4600 & 0.4600 & 0.0600 \\
\hline & $p=0.8$ & 1 & 0 & 0 & 0.4400 & 0.4400 & 0.0400 \\
\hline & $p=0.9$ & 1 & 0 & 0 & 0.4200 & 0.4200 & 0.0200 \\
\hline \multirow{9}{*}{$I_{\Phi_{1, \exp }}$} & $p=0.1$ & 1 & 0 & 0 & 0.2499 & 0.2499 & 0.0183 \\
\hline & $p=0.2$ & 1 & 0 & 0 & 0.2297 & 0.2297 & 0.0143 \\
\hline & $p=0.3$ & 1 & 0 & 0 & 0.2106 & 0.2106 & 0.0108 \\
\hline & $p=0.4$ & 1 & 0 & 0 & 0.1926 & 0.1926 & 0.0078 \\
\hline & $p=0.5$ & 1 & 0 & 0 & 0.1756 & 0.1756 & 0.0053 \\
\hline & $p=0.6$ & 1 & 0 & 0 & 0.1596 & 0.1596 & 0.0034 \\
\hline & $p=0.7$ & 1 & 0 & 0 & 0.1446 & 0.1446 & 0.0019 \\
\hline & $p=0.8$ & 1 & 0 & 0 & 0.1305 & 0.1305 & 0.0008 \\
\hline & $p=0.9$ & 1 & 0 & 0 & 0.1173 & 0.1173 & 0.0002 \\
\hline \multirow{9}{*}{$I_{\Phi_{1, \text { sin }}}$} & $p=0.1$ & 1 & 0 & 0 & 0.7426 & 0.7426 & 0.2126 \\
\hline & $p=0.2$ & 1 & 0 & 0 & 0.7195 & 0.7195 & 0.1864 \\
\hline & $p=0.3$ & 1 & 0 & 0 & 0.6958 & 0.6958 & 0.1607 \\
\hline & $p=0.4$ & 1 & 0 & 0 & 0.6714 & 0.6714 & 0.1356 \\
\hline & $p=0.5$ & 1 & 0 & 0 & 0.6464 & 0.6464 & 0.1111 \\
\hline & $p=0.6$ & 1 & 0 & 0 & 0.6209 & 0.6209 & 0.0873 \\
\hline & $p=0.7$ & 1 & 0 & 0 & 0.5949 & 0.5949 & 0.0642 \\
\hline & $p=0.8$ & 1 & 0 & 0 & 0.5685 & 0.5685 & 0.0419 \\
\hline & $p=0.9$ & 1 & 0 & 0 & 0.5417 & 0.5417 & 0.0205 \\
\hline
\end{tabular}

TABLE 1: IVIF entropies $I_{\Phi_{1, \text { cost }}}, I_{\Phi_{1, \text { exp }}}$, and $I_{\Phi_{1, \text { sin }}}$ of $A_{i}(i=$ $1, \ldots, 6)$.

According to (14), we can construct different entropies on IVIFSs based on various $\Phi$ s. Some example functions that satisfy the previous conditions are presented as follows:

$$
\begin{gathered}
\Phi_{n, \text { cost }}(x, y)=(x+y)^{n}, \quad n=1,2, \ldots, \\
\Phi_{n, \exp }(x, y)=(x+y)^{n} \cdot e^{1-(x+y)}, \quad n=1,2, \ldots, \\
\Phi_{n, \sin }(x, y)=(x+y)^{n} \cdot \sin \left(\frac{\pi}{2}(x+y)\right), \quad n=1,2, \ldots
\end{gathered}
$$

\subsection{Experimental Analysis of IVIF Entropies}

\subsubsection{Examples}

Example 10. Let us assume six IVIFSs, namely, $A_{1}=\{([0,0]$, $[0,0])\}, A_{2}=\{([1,1],[0,0])\}, A_{3}=\{([0.4,0.4],[0.6,0.6])\}$, $A_{4}=\{([0.1,0.2],[0.3,0.4])\}, A_{5}=\{([0.3,0.4],[0.1,0.2])\}$, and $A_{6}=\{([0.3,0.4],[0.5,0.6])\}$ on $X=\{x\}$.

In this example, we adopt $I_{\Phi_{1, \text { cost }}}, I_{\Phi_{1, \text { exp }}}$, and $I_{\Phi_{1, \text { sin }}}$ to calculate the entropies of $A_{i}(i=1, \ldots, 6)$ by choosing different values of parameter $p(p=0.1,0.2, \ldots, 0.9)$. The entropies of $A_{i}(i=1, \ldots, 6)$ are shown in Table 1. 
TABLE 2: IVIF entropies $I_{\Phi_{n, \text { cost }}}, I_{\Phi_{n, \text { exp }}}$, and $I_{\Phi_{n, \text { sin }}}$ of $A_{i}(i=$ $1, \ldots, 6)$.

\begin{tabular}{cccccccc}
\hline & & $A_{1}$ & $A_{2}$ & $A_{3}$ & $A_{4}$ & $A_{5}$ & $A_{6}$ \\
\hline \multirow{4}{*}{$I_{\Phi_{n, \text { cost }}}$} & $n=1$ & 1 & 0 & 0 & 0.5000 & 0.5000 & 0.1000 \\
& $n=2$ & 1 & 0 & 0 & 0.7500 & 0.7500 & 0.1900 \\
& $n=3$ & 1 & 0 & 0 & 0.8750 & 0.8750 & 0.2710 \\
& $n=4$ & 1 & 0 & 0 & 0.9375 & 0.9375 & 0.3439 \\
& $n=5$ & 1 & 0 & 0 & 0.9688 & 0.9688 & 0.4095 \\
\hline \multirow{5}{*}{$I_{\Phi_{n, \text { exp }}}$} & $n=1$ & 1 & 0 & 0 & 0.1756 & 0.1756 & 0.0053 \\
& $n=2$ & 1 & 0 & 0 & 0.5878 & 0.5878 & 0.1048 \\
& $n=3$ & 1 & 0 & 0 & 0.7939 & 0.7939 & 0.1943 \\
& $n=4$ & 1 & 0 & 0 & 0.8970 & 0.8970 & 0.2749 \\
$I_{\Phi_{n, \text { sin }}}$ & $n=5$ & 1 & 0 & 0 & 0.9485 & 0.9485 & 0.3474 \\
& $n=1$ & 1 & 0 & 0 & 0.6464 & 0.6464 & 0.1111 \\
& $n=2$ & 1 & 0 & 0 & 0.8232 & 0.8232 & 0.2000 \\
& $n=3$ & 1 & 0 & 0 & 0.9116 & 0.9116 & 0.2800 \\
& $n=4$ & 1 & 0 & 0 & 0.9558 & 0.9558 & 0.3520 \\
& $n=5$ & 1 & 0 & 0 & 0.9779 & 0.9779 & 0.4168 \\
\hline
\end{tabular}

Example 11. The conditions of this example are the same as Example 10. In this example, we adopt $I_{\Phi_{n, \text { cost }}}, I_{\Phi_{n, \text { exp }}}$, and $I_{\Phi_{n, \text { sin }}}(n=1,2,3,4,5)$ with $p=0.5$ to calculate the entropies of $A_{i}(i=1, \ldots, 6)$. The entropies of $A_{i}(i=1, \ldots, 6)$ are shown in Table 2.

3.4. Analysis of the Experimental Results. The results of Example 10 show that $I_{\Phi_{n, \text { cost }}}, I_{\Phi_{n, \text { exp }}}$, and $I_{\Phi_{n, \text { sin }}}$ are decreasing functions of $p(p \in(0,1))$ and satisfy the four properties of Definition 8. The results of Example 11 show that $I_{\Phi_{n, \text { cost }}}, I_{\Phi_{n, \text { exp }}}$, and $I_{\Phi_{n, \text { sin }}}$ can be regarded as increasing functions of $n(n=$ $1,2, \ldots)$.

\section{MADM Method within the Framework of IVIFS}

In this section, we present a novel method to solve the decision-making problems with unknown attribute weights based on the ranking functions and IVIF entropy. Let $A=\left\{A_{1}\right.$, $\left.A_{2}, \ldots, A_{n}\right\}$ be a set of alternatives and $G=\left\{G_{1}, G_{2}, \ldots, G_{k}\right\}$ be a set of attributes. The IVIF decision matrix $D$ of $A$ on $G$ is

$$
D=\left[\begin{array}{cccc}
\alpha_{11} & \alpha_{12} & \cdots & \alpha_{1 k} \\
\alpha_{21} & \alpha_{22} & \cdots & \alpha_{2 k} \\
\vdots & \vdots & \ddots & \vdots \\
\alpha_{n 1} & \alpha_{n 2} & \cdots & \alpha_{n k}
\end{array}\right]
$$

where $\alpha_{i j}(i=1,2, \ldots, n ; j=1,2, \ldots, k)$ denotes the IVIF numbers. In the following, we introduce our method.

Definition 12. Let $D$ be the IVIF decision-making matrix. We call $I=\left[I_{i j}\right]_{n \times k}$ an IVIF entropy matrix of $D$, where $I_{i j}$ denotes the IVIF entropy of $\alpha_{i j}(i=1,2, \ldots, n ; l=1,2, \ldots, k)$.

Step 1 (determine the attribute weights). Let $\alpha_{i j}=$ $\left(\left[a_{i j}, b_{i j}\right],\left[c_{i j}, d_{i j}\right]\right)(i=1,2, \ldots, n ; j=1,2, \ldots, k)$. We introduce an IVIF entropy matrix. From Definition 12, we obtain the entropy matrix $I=\left[I_{i j}\right]_{n \times k}$ of the decision matrix $D$. We normalize the entropy matrix $I$ to get $\bar{I}=\left[\bar{I}_{i j}\right]_{n \times k}$, where $\bar{I}_{i j}=I_{i j} / \max \left\{I_{1 j}, I_{2 j}, \ldots, I_{n j}\right\}$ for $i=1,2, \ldots, n$ and $j=$ $1,2, \ldots, k$. Finally, the attribute weight $w_{j}(j=1,2, \ldots, k)$ is calculated by

$$
w_{j}=\frac{1-\rho_{j}}{k-\Gamma},
$$

where $\rho_{j}=\sum_{t=1}^{n} \bar{I}_{t j}(j=1,2, \ldots, k)$ and $\Gamma=\sum_{j=1}^{k} \rho_{j}$.

Step 2. Based on the attribute weights obtained in Step 1, the weighted arithmetic average value expressed by $\alpha_{i}=$ $\left(\left[a_{i}, b_{i}\right],\left[c_{i}, d_{i}\right]\right)$ for $A_{i}(i=1,2, \ldots, n)$ is calculated using the IIFWA operator $[29,30]$

$$
\begin{aligned}
\alpha_{i}= & \operatorname{IIFWA}_{w}\left(\alpha_{i 1}, \alpha_{i 2}, \ldots, \alpha_{i k}\right) \\
= & w_{1} \alpha_{i 1} \oplus w_{2} \alpha_{i 2} \oplus \cdots \oplus w_{k} \alpha_{i k} \\
= & \left(\left[1-\prod_{j=1}^{k}\left(1-a_{i j}\right)^{w_{j}}, 1-\prod_{j=1}^{k}\left(1-b_{i j}\right)^{w_{j}}\right],\right. \\
& {\left.\left[\prod_{j=1}^{k} c_{i j}{ }^{w_{j}}, \prod_{j=1}^{k} d_{i j}{ }^{w_{j}}\right]\right), }
\end{aligned}
$$

where $a_{i}=1-\prod_{j=1}^{k}\left(1-a_{i j}\right)^{w_{j}}, b_{i}=1-\prod_{j=1}^{k}\left(1-b_{i j}\right)^{w_{j}}$, $c_{i}=\prod_{j=1}^{k} c_{i j}{ }^{w_{j}}$, and $d_{i}=\prod_{j=1}^{k} d_{i j}{ }^{w_{j}}$.

Step 3. Rank all the alternatives $\alpha_{i}(i=1,2, \ldots, n)$ according to Definition 5 and select the most desirable alternative.

\section{Numerical Examples}

\subsection{Example}

Example 13. This example is adapted from [11]. In this supplier selection problem, five possible alternatives denoted by $A_{i}(i=1,2,3,4,5)$ exist on the following six attributes denoted by $G_{j}(j=1,2,3,4,5,6)$. The ratings of the alternatives are given in the IVIF decision matrix shown in Tables 3 and 4 .

In this example, we adopt $I_{\Phi_{n, \text { cost }}}(n=1,10,100 ; p=0.1$, $0.5,0.9)$ to solve the supplier selection problem. The decisionmaking process is as follows.

Step 1. Based on $I_{\Phi_{n, \text { cost }}}(n=1,10,100 ; p=0.1,0.5,0.9)$, the attribute weights are obtained, as shown in Table 5.

Step 2. The weighted arithmetic average values $\alpha_{i}$ for $A_{i}(i=$ $1,2,3,4,5)$ are calculated.

Step 3. The score values $s\left(\alpha_{i}\right)(i=1,2,3,4,5)$ are calculated, and the decision-making results are shown in Table 6.

5.2. Experimental Analysis. Similarly, we adopt $I_{\Phi_{n, \exp }}$ and $I_{\Phi_{n, \text { sin }}}(n=1,10,100 ; p=0.1,0.5,0.9)$ to implement the decision-making process for this supplier selection problem. 
TABLE 3: IVIF decision matrix $D$.

\begin{tabular}{lcrr}
\hline & $X_{1}$ & $X_{2}$ & $X_{3}$ \\
\hline$A_{1}$ & $([0.2,0.5],[0.4,0.5])$ & $([0.3,0.4],[0.3,0.4])$ & $([0.3,0.4],[0.4,0.6])$ \\
$A_{2}$ & $([0.3,0.4],[0.3,0.5])$ & $([0.5,0.8],[0.1,0.2])$ & $([0.5,0.6],[0.2,0.4])$ \\
$A_{3}$ & $([0.4,0.5],[0.2,0.3])$ & $([0.7,0.8],[0.0,0.1])$ & $([0.6,0.7],[0.1,0.3])$ \\
$A_{4}$ & $([0.4,0.7],[0.0,0.2])$ & $([0.9,1.0],[0.0,0.0])$ & $([0.7,0.8],[0.0,0.1])$ \\
$A_{5}$ & $([0.6,0.7],[0.1,0.3])$ & $([0.4,0.6],[0.1,0.2])$ & $([0.7,0.8],[0.0,0.1])$ \\
\hline
\end{tabular}

TABLE 4: IVIF decision matrix $D$.

\begin{tabular}{lcrr}
\hline & $X_{4}$ & $X_{5}$ & $X_{6}$ \\
\hline$A_{1}$ & $([0.3,0.5],[0.4,0.5])$ & $([0.2,0.4],[0.5,0.6])$ & $([0.3,0.4],[0.4,0.5])$ \\
$A_{2}$ & $([0.5,0.6],[0.2,0.3])$ & $([0.4,0.6],[0.3,0.4])$ & $([0.6,0.7],[0.1,0.3])$ \\
$A_{3}$ & $([0.7,0.8],[0.1,0.2])$ & $([0.3,0.4],[0.4,0.5])$ & $([0.5,0.6],[0.1,0.2])$ \\
$A_{4}$ & $([0.8,0.9],[0.0,0.1])$ & $([0.5,0.7],[0.2,0.3])$ & $([0.6,0.8],[0.1,0.2])$ \\
$A_{5}$ & $([0.3,0.4],[0.2,0.3])$ & $([0.8,0.9],[0.0,0.1])$ & $([0.4,0.5],[0.3,0.4])$ \\
\hline
\end{tabular}

TABLE 5: Attributes weights based on $I_{\Phi_{n, \text { cost }}}(n=1,10,100 ; p=0.1,0.5,0.9)$.

\begin{tabular}{lccccc}
\hline Entropy & $w_{1}$ & $w_{2}$ & $w_{3}$ & $w_{4}$ & $w_{5}$ \\
\hline$\left.I_{\Phi_{n, \text { cost }}}\right|_{p=0.1, n=1}$ & 0.1468 & 0.1382 & 0.2278 & 0.1109 & 0.2069 \\
$\left.I_{\Phi_{n, \text { cost }}}\right|_{p=0.5, n=1}$ & 0.1783 & 0.1486 & 0.2253 & 0.0953 & 0.1907 \\
$\left.I_{\Phi_{n, \text { cost }}}\right|_{p=0.9, n=1}$ & 0.2011 & 0.2144 & 0.2212 & 0.0751 & 0.1159 \\
$\left.I_{\Phi_{n, \text { cost }}}\right|_{p=0.1, n=10}$ & 0.1718 & 0.1556 & 0.1741 & 0.1596 & 0.1696 \\
$\left.I_{\Phi_{n, \text { cost }}}\right|_{p=0.5, n=10}$ & 0.1786 & 0.1562 & 0.1805 & 0.1472 & 0.1673 \\
$\left.I_{\Phi_{n, \text { cost }}}\right|_{p=0.9, n=10}$ & 0.2034 & 0.1934 & 0.1812 & 0.1195 & 0.1724 \\
$\left.I_{\Phi_{n, \text { cost }}}\right|_{p=0.1, n=100}$ & 0.1667 & 0.1667 & 0.1667 & 0.1667 & 0.1693 \\
$\left.I_{\Phi_{n, \text { cost }}}\right|_{p=0.5, n=100}$ & 0.1667 & 0.1665 & 0.1667 & 0.1667 & 0.1667 \\
$\left.I_{\Phi_{n, \text { cost }}}\right|_{p=0.9, n=100}$ & 0.1732 & 0.1592 & 0.1697 & 0.1622 & 0.1667 \\
\hline
\end{tabular}

TABLE 6: Scores values $s\left(\alpha_{i}\right)(i=1,2,3,4,5)$.

\begin{tabular}{lcccccc}
\hline Entropy & $s\left(\alpha_{1}\right)$ & $s\left(\alpha_{2}\right)$ & $s\left(\alpha_{3}\right)$ & $s\left(\alpha_{4}\right)$ & $s\left(\alpha_{5}\right)$ & Ranking order of $A_{i}(i=1,2,3,4,5)$ \\
\hline$\left.I_{\Phi_{n, \text { cost }}}\right|_{p=0.1, n=1}$ & -0.1168 & 0.2872 & 0.4647 & 0.8408 & 0.5785 & $A_{4}>A_{5}>A_{3}>A_{2}>A_{1}$ \\
$\left.I_{\Phi_{n, \text { cost }}}\right|_{p=0.5, n=1}$ & -0.1139 & 0.2817 & 0.4643 & 0.8398 & 0.5754 & $A_{4}>A_{5}>A_{3}>A_{2}>A_{1}$ \\
$\left.I_{\Phi_{n, \text { cost }}}\right|_{p=0.9, n=1}$ & -0.0968 & 0.3042 & 0.4965 & 0.8526 & 0.5412 & $A_{4}>A_{5}>A_{3}>A_{2}>A_{1}$ \\
$\left.I_{\Phi_{n, \text { cost }}}\right|_{p=0.1, n=10}$ & -0.1044 & 0.2922 & 0.4855 & 0.8472 & 0.5399 & $A_{4}>A_{5}>A_{3}>A_{2}>A_{1}$ \\
$\left.I_{\Phi_{n, \text { cost }}}\right|_{p=0.5, n=10}$ & -0.1050 & 0.2905 & 0.4829 & 0.8459 & 0.5429 & $A_{4}>A_{5}>A_{3}>A_{2}>A_{1}$ \\
$\left.I_{\Phi_{n, \text { cost }}}\right|_{p=0.9, n=10}$ & -0.0951 & 0.3024 & 0.4977 & 0.8510 & 0.5213 & $A_{4}>A_{5}>A_{3}>A_{2}>A_{1}$ \\
$\left.I_{\Phi_{n, \text { cost }}}\right|_{p=0.1, n=100}$ & -0.1021 & 0.2968 & 0.4917 & 0.8503 & 0.5349 & $A_{4}>A_{5}>A_{3}>A_{2}>A_{1}$ \\
$\left.I_{\Phi_{n, \text { cost }}}\right|_{p=0.5, n=100}$ & -0.1021 & 0.2967 & 0.4916 & 0.8503 & 0.5349 & $A_{4}>A_{5}>A_{3}>A_{2}>A_{1}$ \\
$\left.I_{\Phi_{n, \text { cost }}}\right|_{p=0.9, n=100}$ & -0.1029 & 0.2941 & 0.4883 & 0.8481 & 0.5351 & $A_{4}>A_{5}>A_{3}>A_{2}>A_{1}$ \\
\hline
\end{tabular}

All the ranking results are the same as the ranking order of $I_{\Phi_{n, \text { cost }}}(n=1,10,100 ; p=0.1,0.5,0.9)$, that is, $A_{4}>A_{5}>$ $A_{3}>A_{2}>A_{1}$. By applying the methods in [11, 41] to Example 13, the ranking order is $A_{4}>A_{5}>A_{3}>A_{2}>A_{1}$, and the most desirable one is $A_{4}$ which is identical to ours. From the aforementioned results, the proposed decisionmaking method in this paper can be suitably utilized to solve the IVIF MADM problem with completely unknown attribute weights. Different IVIF entropies clearly offer various iuchoices for assessing the attribute weights.

\section{Conclusion}

In this paper, we have focused on solving the IVIF MADM problem with completely unknown attribute weights. We first introduced a new definition of IVIF entropy and some calculation methods of different IVIF entropies. Subsequently, we proposed an entropy-based MADM method in the framework of IVIFS. The proposed method can be utilized to solve fuzzy and uncertain decision-making problems derived from supplier selection, public risk, medical diagnosis, and other problems in any aspects. 


\section{Acknowledgments}

The authors is very grateful to the anonymous referees and editors, for their constructive comments and suggestions that have led to an improved version of this paper. This research was supported by the Fundamental Research Funds for the Central Universities under Grant no. 2013JBM023.

\section{References}

[1] K. T. Atanassov, "Intuitionistic fuzzy sets," Fuzzy Sets and Systems, vol. 20, no. 1, pp. 87-96, 1986.

[2] K. Atanassov and G. Gargov, "Interval valued intuitionistic fuzzy sets," Fuzzy Sets and Systems, vol. 31, no. 3, pp. 343-349, 1989.

[3] K. Atanassov and C. Georgiev, "Intuitionistic fuzzy prolog," Fuzzy Sets and Systems, vol. 53, no. 2, pp. 121-128, 1993.

[4] T. Chaira, "Intuitionistic fuzzy segmentation of medical images," IEEE Transactions on Biomedical Engineering, vol. 57, no. 6, pp. 1430-1436, 2010.

[5] T. Chaira, "A novel intuitionistic fuzzy $\mathrm{C}$ means clustering algorithm and its application to medical images," Applied Soft Computing Journal, vol. 11, no. 2, pp. 1711-1717, 2011.

[6] T. Chaira and A. K. Ray, "A new measure using intuitionistic fuzzy set theory and its application to edge detection," Applied Soft Computing Journal, vol. 8, no. 2, pp. 919-927, 2008.

[7] C. Cornelis, G. Deschrijver, and E. E. Kerre, "Implication in intuitionistic fuzzy and interval-valued fuzzy set theory: construction, classification, application," International Journal of Approximate Reasoning, vol. 35, no. 1, pp. 55-95, 2004.

[8] W.-L. Hung and M.-S. Yang, "On the J-divergence of intuitionistic fuzzy sets with its application to pattern recognition," Information Sciences, vol. 178, no. 6, pp. 1641-1650, 2008.

[9] I. K. Vlachos and G. D. Sergiadis, "Intuitionistic fuzzy information-applications to pattern recognition," Pattern Recognition Letters, vol. 28, no. 2, pp. 197-206, 2007.

[10] Z. Wang, Z. Xu, S. Liu, and J. Tang, "A netting clustering analysis method under intuitionistic fuzzy environment," Applied Soft Computing Journal, vol. 11, no. 8, pp. 5558-5564, 2011.

[11] Z. S. Xu, Intuitionistic Fuzzy Information Aggregation Theory and Application, Science Press, Beijing, China, 2008.

[12] Z. S. Xu, J. Chen, and J. Wu, "Clustering algorithm for intuitionistic fuzzy sets," Information Sciences, vol. 178, no. 19, pp. 37753790, 2008

[13] T.-Y. Chen, "Bivariate models of optimism and pessimism in multi-criteria decision-making based on intuitionistic fuzzy sets," Information Sciences, vol. 181, no. 11, pp. 2139-2165, 2011.

[14] T.-Y. Chen and C.-H. Li, "Determining objective weights with intuitionistic fuzzy entropy measures: a comparative analysis," Information Sciences, vol. 180, no. 21, pp. 4207-4222, 2010.

[15] D. Dubey, S. Chandra, and A. Mehra, "Fuzzy linear programming under interval uncertainty based on IFS representation," Fuzzy Sets and Systems, vol. 188, no. 1, pp. 68-87, 2012.

[16] C. L. Hwang and M. J. Lin, Group Decision Making under Multiple Criteria: Methods and Applications, Springer, Berlin, Germany, 1987.

[17] E. K. Zavadskas, Z. Turskis, L. Ustinovichius, and G. Shevchenko, "Attributes weights determining peculiarities in multiple attribute decision making methods," Engineering Economics, no. 1, pp. 32-43, 2010.
[18] S.-M. Chen and J.-M. Tan, "Handling multicriteria fuzzy decision-making problems based on vague set theory," Fuzzy Sets and Systems, vol. 67, no. 2, pp. 163-172, 1994.

[19] Z. S. Xu, "A method based on distance measure for intervalvalued intuitionistic fuzzy group decision making," Information Sciences, vol. 180, no. 1, pp. 181-190, 2010.

[20] J. Ye, "Multicriteria fuzzy decision-making method based on a novel accuracy function under interval-valued intuitionistic fuzzy environment," Expert Systems with Applications, vol. 36, no. 3, pp. 6899-6902, 2009.

[21] V. Lakshmana Gomathi Nayagam, S. Muralikrishnan, and G. Sivaraman, "Multi-criteria decision-making method based on interval-valued intuitionistic fuzzy sets," Expert Systems with Applications, vol. 38, no. 3, pp. 1464-1467, 2011.

[22] Z. Wang, K. W. Li, and W. Wang, "An approach to multiattribute decision making with interval-valued intuitionistic fuzzy assessments and incomplete weights," Information Sciences, vol. 179, no. 17, pp. 3026-3040, 2009.

[23] K. T. Atanassov, "Operators over interval valued intuitionistic fuzzy sets," Fuzzy Sets and Systems, vol. 64, no. 2, pp. 159-174, 1994.

[24] G. Beliakov, H. Bustince, D. P. Goswami, U. K. Mukherjee, and N. R. Pal, "On averaging operators for Atanassov's intuitionistic fuzzy sets," Information Sciences, vol. 181, no. 6, pp. 1116-1124, 2011.

[25] D.-F. Li, “The GOWA operator based approach to multiattribute decision making using intuitionistic fuzzy sets," Mathematical and Computer Modelling, vol. 53, no. 5-6, pp. 1182-1196, 2011.

[26] Z.-X. Su, G.-P. Xia, M.-Y. Chen, and L. Wang, "Induced generalized intuitionistic fuzzy OWA operator for multi-attribute group decision making," Expert Systems with Applications, vol. 39, no. 2, pp. 1902-1910, 2012.

[27] C. Tan and X. Chen, "Intuitionistic fuzzy Choquet integral operator for multi-criteria decision making," Expert Systems with Applications, vol. 37, no. 1, pp. 149-157, 2010.

[28] G. Wei, "Some induced geometric aggregation operators with intuitionistic fuzzy information and their application to group decision making," Applied Soft Computing Journal, vol. 10, no. 2, pp. 423-431, 2010.

[29] Z. S. Xu, "Approaches to multiple attribute group decision making based on intuitionistic fuzzy power aggregation operators," Knowledge-Based Systems, vol. 24, no. 6, pp. 749-760, 2011.

[30] Z. S. Xu, "Intuitionistic fuzzy aggregation operators," IEEE Transactions on Fuzzy Systems, vol. 15, no. 6, pp. 1179-1187, 2007.

[31] Z. S. Xu and M. Xia, "Induced generalized intuitionistic fuzzy operators," Knowledge-Based Systems, vol. 24, no. 2, pp. 197-209, 2011.

[32] S. Zeng and W. Su, "Intuitionistic fuzzy ordered weighted distance operator," Knowledge-Based Systems, vol. 24, no. 8, pp. 1224-1232, 2011.

[33] L. Zhou and W.-Z. Wu, "On generalized intuitionistic fuzzy rough approximation operators," Information Sciences, vol. 178, no. 11, pp. 2448-2465, 2008.

[34] Z. S. Xu and R. R. Yager, "Dynamic intuitionistic fuzzy multiattribute decision making," International Journal of Approximate Reasoning, vol. 48, no. 1, pp. 246-262, 2008.

[35] D.-F. Li, "Closeness coefficient based nonlinear programming method for interval-valued intuitionistic fuzzy multiattribute decision making with incomplete preference information," Applied Soft Computing Journal, vol. 11, no. 4, pp. 3402-3418, 2011. 
[36] D.-F. Li, "Linear programming method for MADM with interval-valued intuitionistic fuzzy sets," Expert Systems with Applications, vol. 37, no. 8, pp. 5939-5945, 2010.

[37] D.-F. Li, “TOPSIS-based nonlinear-programming methodology for multiattribute decision making with interval-valued intuitionistic fuzzy sets," IEEE Transactions on Fuzzy Systems, vol. 18, no. 2, pp. 299-311, 2010.

[38] D.-F. Li, Y.-C. Wang, S. Liu, and F. Shan, "Fractional programming methodology for multi-attribute group decision-making using IFS," Applied Soft Computing Journal, vol. 9, no. 1, pp. 219225, 2009.

[39] D. G. Park, Y. C. Kwun, J. H. Park, and I. Y. Park, "Correlation coefficient of interval-valued intuitionistic fuzzy sets and its application to multiple attribute group decision making problems," Mathematical and Computer Modelling, vol. 50, no. 9-10, pp. 1279-1293, 2009.

[40] M. Xia and Z. Xu, "Entropy/cross entropy-based group decision making under intuitionistic fuzzy environment," Information Fusion, vol. 13, no. 1, pp. 31-47, 2012.

[41] J. Ye, "Multicriteria fuzzy decision-making method using entropy weights-based correlation coefficients of intervalvalued intuitionistic fuzzy sets," Applied Mathematical Modelling, vol. 34, no. 12, pp. 3864-3870, 2010.

[42] T. L. Saaty, The Analytic Hierarchy Process, McGraw-Hill, New York, NY, USA, 1980.

[43] P. Burillo and H. Bustince, "Entropy on intuitionistic fuzzy sets and on interval-valued fuzzy sets," Fuzzy Sets and Systems, vol. 78, no. 3, pp. 305-316, 1996.

[44] E. Szmidt and J. Kacprzyk, "Entropy for intuitionistic fuzzy sets," Fuzzy Sets and Systems, vol. 118, no. 3, pp. 467-477, 2001.

[45] W.-L. Hung, "A note on entropy of intuitionistic fuzzy sets," International Journal of Uncertainty, Fuzziness and KnowlegeBased Systems, vol. 11, no. 5, pp. 627-633, 2003.

[46] H. Zhang, W. Zhang, and C. Mei, "Entropy of interval-valued fuzzy sets based on distance and its relationship with similarity measure," Knowledge-Based Systems, vol. 22, no. 6, pp. 449-454, 2009.

[47] J. Ye, "Two effective measures of intuitionistic fuzzy entropy," Computing, vol. 87, no. 1-2, pp. 55-62, 2010.

[48] L. A. Zadeh, "Fuzzy sets and systems," in Proceedings of the Symposium on Systems Theory, pp. 29-37, Polytechnic Institute of Brooklyn, New York, NY, USA, 1965.

[49] Q.-S. Zhang, S. Jiang, B. Jia, and S. Luo, "Some information measures for interval-valued intuitionistic fuzzy sets," Information Sciences, vol. 180, no. 24, pp. 5130-5145, 2010.

[50] H. Bustince, "Construction of intuitionistic fuzzy relations with predetermined properties," Fuzzy Sets and Systems, vol. 109, no. 3, pp. 379-403, 2000.

[51] H. Bustince and P. Burillo, "Correlation of interval-valued intuitionistic fuzzy sets," Fuzzy Sets and Systems, vol. 74, no. 2, pp. 237-244, 1995. 


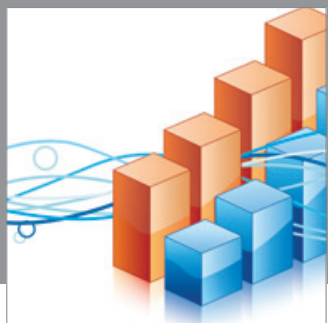

Advances in

Operations Research

mansans

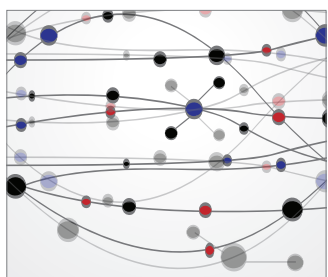

The Scientific World Journal
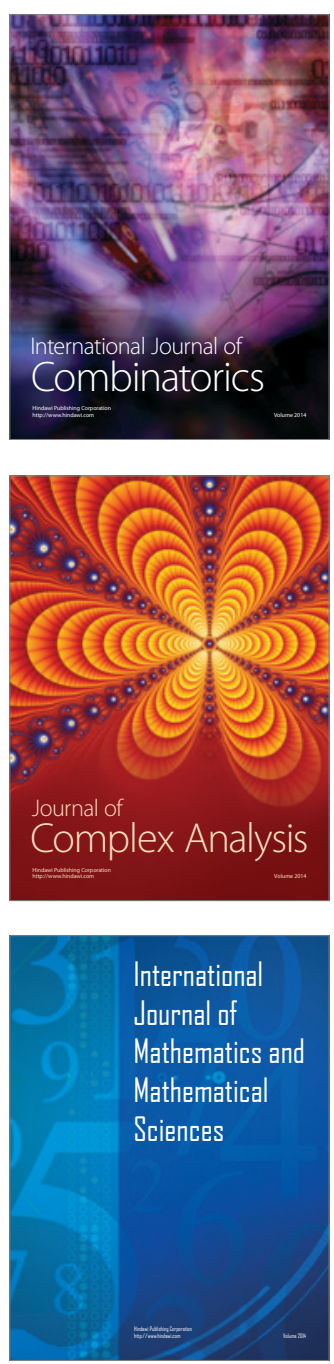
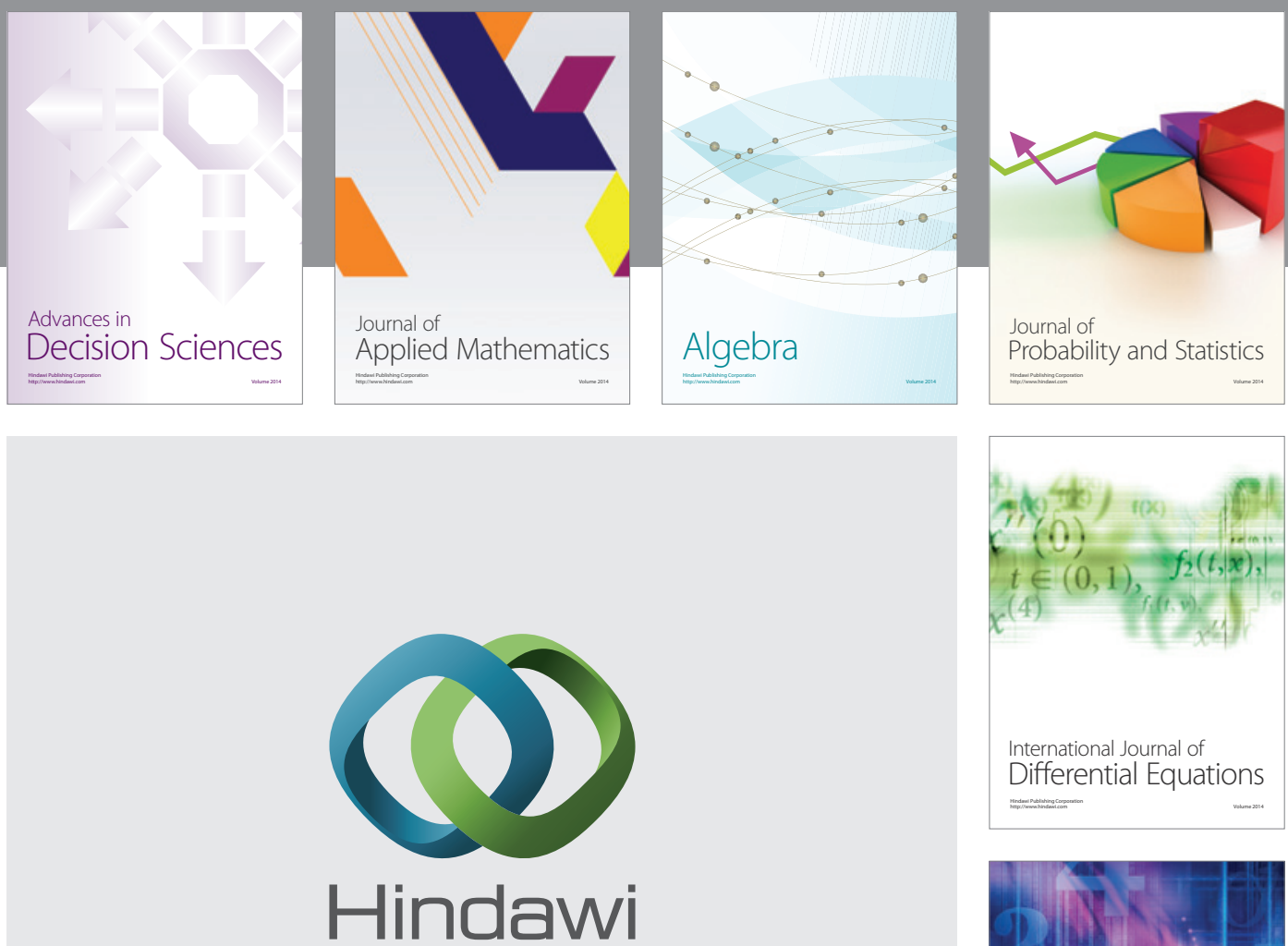

Submit your manuscripts at http://www.hindawi.com
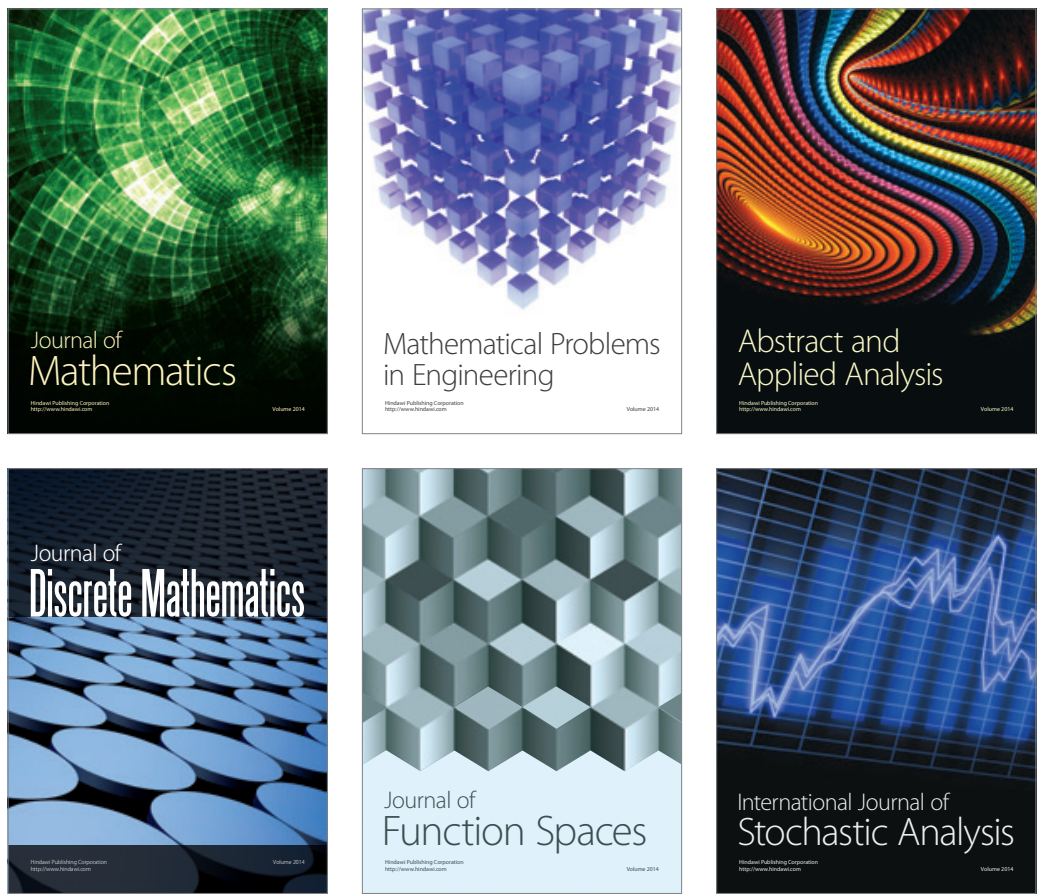

Journal of

Function Spaces

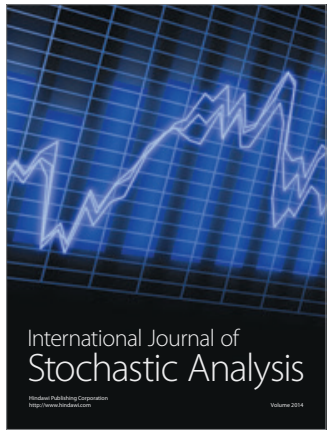

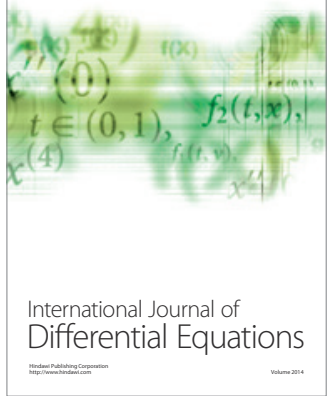
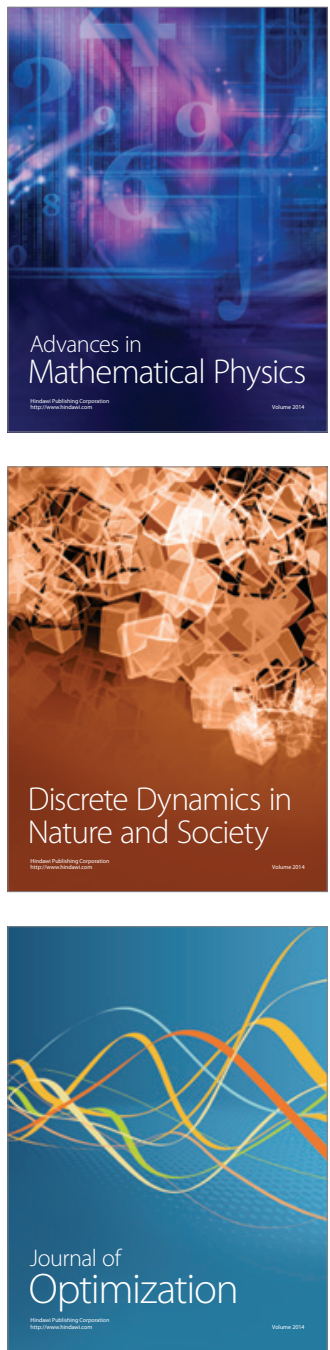